\title{
A modelação aplicada à temática dos riscos naturais: 0 caso concreto de instituições de ensino superior em Portugal
}

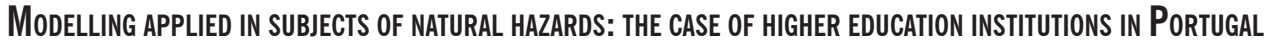 \\ Sara Batista Gomes Moutinho ${ }^{1}$, Rui Miguel Marques Moura², Clara Maria da Silva Vasconcelos ${ }^{3}$ \\ 1- Instituto de Ciências da Terra/Faculdade de Ciências/Universidade do Porto. sara.moutinho@fc.up.pt \\ 2- Instituto de Ciências da Terra/Faculdade de Ciências/Universidade do Porto.rmmoura@fc.up.pt \\ 3- Instituto de Ciências da Terra /Faculdade de Ciências/Universidade do Porto. csvascon@fc.up.pt
}

ABSTRACT: The use of modelling as a methodology to aid in building mental models is very important in the process of recreating and simulating natural phenomena, in particular geological and environmental phenomena. In order to check whether the use of modelling is significant as a methodology for the success of student learning of natural hazards in higher education, this study included all Portuguese public university syllabi that address natural hazards. Only graduation syllabi were selected for this study. Eight graduation syllabi were analyzed, through an analysis screen, conducted in seven Portuguese universities. The evidence suggests that none of the syllabi in this analysis entail the use of modeling as a teaching strategy for natural hazards subjects, while an essentially traditional method is predominantly used. The need for intervention regarding teaching methodologies that value the role of models and modeling in higher education is highlighted.

\section{Manuscrito:}

Recebido: 29/09/2015

Corrigido: $15 / 02 / 2016$

Aceito: 15/03/2016

Citation: Moutinho S.B.G., Moura R.M.M., Vasconcelos C.M.S. 2016. A modelação aplicada à temática dos riscos naturais: 0 caso concreto de instituições de ensino superior em Portugal Terræ Didatica, 12(2):118-125. <http://www.ige.unicamp.br/terraedidatica/>

Keywords: Modelling, university education, natural hazards, syllabus.

\section{Introdução}

A psicologia cognitiva é uma disciplina fundamental ao nível da educação em ciências, pois tem dado grandes contributos para a compreensão do processo de aprendizagem, não havendo dúvidas quanto à sua importância e o seu papel ao nível dos estudos relacionados com a educação (Greca \& Moreira 2000). Atualmente defende-se que o ensino das ciências deve potencializar uma aprendizagem significativa, considerando-se fundamental o recurso a metodologias que promovam o desenvolvimento do conhecimento científico dos estudantes. A escola é vista como um lugar de aprendizagem, ou seja, de mudanças relativamente permanentes na conduta e no conhecimento dos sujeitos, como resultado da experiência e dos processos de associação e condicionamento (Alves 2014). A psicologia cognitiva explica que a forma como os sujeitos compreendem o mundo depende daquilo que nele existe e, daquilo que compõe a mente humana (Johnson-Laird 1983), admitindo-se que esta tem a capacidade de processar informa- ção e interpretar o mundo através da manipulação de símbolos (Palmero 2008). Este processo resulta no desenvolvimento de modelos mentais dos sujeitos, que lhes permitem compreender o mundo em que vivem e dar resposta a situações do cotidiano com que são confrontados.

A Aprendizagem Baseada em Modelos (ModelBased Learning) é uma metodologia que promove a aprendizagem dos conteúdos científicos através da construção e utilização de modelos, que recriam fenómenos físicos, permitindo desenvolver o conhecimento científico dos estudantes e a sua literacia científica (Gobert \& Buckley 2000), aproximando o processo de aprendizagem dos sujeitos à forma como estes desenvolvem os seus conhecimentos de forma inata, ou seja, os modelos mentais. Esta metodologia promove o contato dos estudantes com modelos, a sua manipulação e comparação com os seus modelos mentais. Desta forma, são geradas as condições necessárias para que se desenvolva uma aprendizagem significativa, através da restruturação das estruturas cognitivas 
dos estudantes (Louca et al. 2011, Palmero 2008).

No caso concreto do ensino das Geociências, a modelação ${ }^{1}$ apresenta-se como uma ferramenta fulcral pois, a recriação dos fenómenos geológicos através de modelos pode reduzir milhões de anos a algumas horas ou minutos (Bolacha et al. 2011, Deus et al. 2011), e permite recriar fenómenos que não são facilmente observáveis num contexto real, como é o caso dos riscos naturais. Assim, se os estudantes forem capazes de manipular, testar e avaliar os modelos, bem como as suas dinâmicas, poderão também aumentar o seu interesse e compreensão sobre as mudanças reais que ocorreram no curso da história da Terra (Deus et al. 2011).

Comprovada a sua importância e utilidade ao nível do ensino das ciências, pretende-se averiguar se a modelação é referida como uma metodologia utilizada no ensino superior para lecionar temáticas de riscos naturais. Este estudo constitui o ponto de partida para o desenvolvimento de um programa de intervenção, que será aplicado a estudantes do ensino superior português, com o objetivo de aplicar e testar vários tipos de modelos na lecionação de temáticas de riscos naturais. Para isso, foi feito o levantamento de todas as unidades curriculares do primeiro ciclo de estudos (licenciatura) que contemplam as temáticas supracitadas, tendo-se obtido uma amostra de oito unidades curriculares. Posteriormente foram analisadas as fichas das unidades curriculares que constituíam a amostra, de acordo com uma grade de análise construída e validada para o efeito por três especialistas com experiência na docência do ensino superior. A elaboração da grade de análise e a definição das categorias e subcategorias de análise foi feita tendo por base uma análise transversal dos conteúdos das várias fichas das unidades curriculares e sintetização dos parâmetros/objetivos mais valorizados nas várias instituições do ensino superior.

\section{Enquadramento Teórico}

\section{Importância dos Modelos no processo de aprendizagem}

Apesar de se reconhecer que a aprendizagem significativa desempenha um papel fulcral no ensino das ciências, em algumas instituições as teorias e modelos científicos ainda são ensinados como verdades, como definitivos e acabados. A literatura

10 termo modelação a que é feito referência no corpo do artigo é equivalente ao termo modelagem, mais comumente utilizado no Brasil. da especialidade refere que os alunos são construtores do seu próprio conhecimento e defende que os professores devem ser capazes de fazer a mediação necessária para a reconstrução interna dos conhecimentos científicos que são externamente construídos. Contudo, os conteúdos científicos não são apresentados aos estudantes como construções científicas. É certo que há vários educadores que promovem o ensino da ciência através de uma perspetiva científica, no entanto, atualmente ainda predomina o ensino tradicional, das teorias definitivas e acabadas (Moreira 2014).

O primeiro passo para o abandono desta corrente consiste em compreender como é que a mente humana se organiza, e como são gerados os conhecimentos dos sujeitos. É nesta perspetiva que surgem os modelos mentais, nos quais se reconhece elevada importância ao nível do desenvolvimento da aprendizagem significativa, pelo facto de auxiliarem o processo de representação do mundo real. Desta forma, importa esclarecer o que se entende por modelo mental, e distingui-los de outros tipos de modelos igualmente importantes no processo de desenvolvimento cognitivo dos estudantes.

O modelo mental é um modelo pessoal, desenvolvido pela mente humana para representar o mundo exterior (Moreira 1996), e que segundo Krapas, Queiroz e Colinvaux (1997), pode ser expresso de várias formas, como por intermédio de ações, da fala, da escrita e do desenho. São modelos extremamente úteis e funcionais para os estudantes, que se socorrem deles para resolver situações-problema, constituindo por isso o seu conhecimento prévio, embora sejam cientificamente inconsistentes (Greca \& Moreira 2000).

Segundo a Teoria dos Modelos Mentais de Johnson-Laird, os modelos mentais são análogos estruturais do mundo, e caraterizam-se por serem simples e representarem sempre entidades concretas (Johnson-Laird 1983). Por poderem ser restruturados pelo sujeito à medida que contactam com outros modelos são dinâmicos e flexíveis, mas são também limitados, porque a sua construção e manipulação depende da memória de trabalho da mente humana (Palmero 2008). Em suma, são uma forma de representação analógica do conhecimento, uma vez que existe uma correspondência direta entre entidades e relações presentes na estrutura dessa representação, e as entidades e relações que se pretende representar (Moreira 2014).

Ao contrário dos modelos mentais, os modelos científicos resultam da criação específica dos 
cientistas para representar uma ideia, um objeto, acontecimento, processo ou sistema (Justi 2006). Por outras palavras, correspondem a modelos conceptuais cientificamente aceites, que traduzem a representação da forma de pensar dos cientistas. Como são geralmente complexos necessitam de simplificação a fim de poderem ser ensinados nas salas de aula de ciências. Estas simplificações são designadas por modelos curriculares. No fundo, estes modelos resultam de adaptações dos modelos científicos, que apesar de mais simples, porque representam apenas uma parte do fenómeno ou das variáveis em estudo, são cientificamente corretos porque têm origem nos modelos científicos.

No ensino das ciências, o professor pretende, por meio de modelos curriculares, que os estudantes reestruturem os seus modelos mentais, com vista a tornarem-se consistentes, e aproximarem-se, progressivamente, dos modelos científicos que, recriam os fenómenos naturais. Assim se compreende que a mente humana opera não só através de modelos mentais, mas também através de modelos conceptuais e teóricos, que podem auxiliar a reestruturação dos modelos mentais, para que estes se tornem congruentes com o conhecimento aceite (modelo científico) numa determinada área científica (Moreira 2014).

Além dos modelos curriculares, Justi (2006) refere que é necessário que o professor recorra a modelos para o ensino, construídos com o propósito de ajudar os alunos a aprender alguns aspetos de um determinado modelo curricular, constituindo representações aproximadas de apenas uma parte da realidade. Estes modelos para o ensino são muito importantes pelo auxílio que prestam aos estudantes na compreensão dos modelos curriculares, ou seja, no seu processo de aprendizagem da ciência.

Não obstante a importância e a utilidade dos vários tipos de modelos, cabe aos docentes a difícil tarefa de adaptar e articular os modelos para o ensino com as temáticas a lecionar em sala de aula. Pelo exposto, facilmente se explica o papel fundamental que o professor possui no processo de aprendizagem, pois deve promover a evolução dos modelos mentais dos estudantes, tornando-os congruentes com o conhecimento científico. $\mathrm{O}$ processo de restruturação dos modelos mentais dos estudantes deve ocorrer de forma natural e espontânea, através de metodologias que os levem a avaliar a importância dos modelos e a relacioná-los com o seu conhecimento prévio (Palmero 2008). Contudo, este processo pode mostrar-se extremamente difícil, devido a um conjunto de variáveis que podem intervir negativamente: (i) a dificuldade em observar o modelo que se pretende lecionar; (ii) os conflitos que podem surgir entre os modelos e a linguagem usada pelo professor e os modelos mentais dos estudantes; (iii) e os conceitos e o vocabulário que os estudantes reconhecem através do uso da linguagem natural, são alguns entraves à restruturação dos seus modelos mentais (Clement 2000).

Além disso, ao contactar com os modelos curriculares em ambiente de sala de aula, os estudantes podem apresentar diferentes comportamentos: (i) tentar interpretá-los de acordo com o seu conhecimento prévio, ou seja, os seus modelos mentais, gerando modelos híbridos; (ii) tentar memorizá-los de forma descontextualizada, através de representações proposicionais internas soltas, resultando numa aprendizagem mecânica; (iii) ou reestruturar os seus modelos mentais tornando-os consistentes com os modelos curriculares (Greca \& Moreira 2000).

\section{Modelação no ensino das ciências (geociências)}

Por se atribuir particular importância ao desenvolvimento cognitivo dos estudantes e à construção dos seus conhecimentos, é atribuída grande importância à Aprendizagem Baseada em Modelos enquanto metodologia de ensino fundamental para explicar conceitos de ciência abstratos e não-observáveis (Treagust et al. 2002).

$\mathrm{O}$ uso de modelos e da modelação remonta ao século XIX, quando se começaram a reproduzir em laboratório alguns fenómenos geológicos (Brandstetter 2011, Graveleau et al. 2012). Nesta altura, o principal papel da modelação era facilitar a reprodução da evolução das causas e dos mecanismos dos processos geológicos (Graveleau et al. 2012), responsáveis pelas estruturas observadas na natureza. Assim, os modelos construídos permitiam aos cientistas desenvolver conhecimentos sobre os processos geológicos e as condições que levavam à sua ocorrência. Contudo, o recurso a esta metodologia foi largamente contestado, não só por questões de representatividade, mas também pelo facto de não conseguir comprovar a ocorrência de um fenómeno na natureza, apesar de demonstrar que uma causa proposta pode produzir um efeito observável (Torres \& Vasconcelos 2014).

Posteriormente, a modelação começou a ser utilizada no ensino da Geologia, inicialmente para 
testar teorias e hipóteses (Brandstetter 2011). Ao ser reconhecida a sua elevada potencialidade como ferramenta auxiliadora do processo de construção do conhecimento (Barbosa 2009), ela passou a ser utilizada no ensino das ciências como metodologia de ensino. Desde então se defende o recurso à modelação nas aulas de ciências por se considerar que o processo de aprendizagem dos estudantes deve ser autónomo e dinâmico. Admite-se assim que os estudantes possuem o seu próprio modelo mental dos modelos curriculares, que podem ser cientificamente inconsistentes e dificultarem o processo de construção do conhecimento, ou até mesmo gerarem conceções alternativas (Treagust et al. 2002, Moutinho et al. 2013). Por isso, a modelação enquanto metodologia de ensino, desempenha um papel relevante na construção do conhecimento científico, na medida em que promove a compreensão da dinâmica dos processos naturais e as variáveis que nele intervêm (Bolacha et al. 2011). Além disso, auxilia o processo de restruturação dos modelos mentais dos estudantes, procurando que se tornem mais consistentes com os modelos curriculares apresentados pelos professores.

Contudo, geralmente as instituições de ensino não promovem a relação entre a prática pedagógica e a proposta educativa, acabando por tratar os conteúdos científicos sem aprofundamento e proximidade da realidade dos alunos. Assim, torna-se urgente contextualizar o ensino das ciências com metodologias que desafiem os estudantes, integrando-os no processo de ensino-aprendizagem de forma ativa, interativa e promovendo o questionamento (Alves 2014).

Reconhece-se elevada potencialidade no ensino suportado nesta metodologia, que possibilita não só a construção do conhecimento, mas também a promoção da literacia científica dos estudantes (Gobert \& Buckley 2000), na medida em que permite aprender ciência, aprender sobre ciência e, aprender como fazer ciência (Justi \& Gilbert 2002a).

Segundo Hodson (1992) aprender ciência implica entender as ideias desenvolvidas pela ciência, constituindo a aprendizagem dos conceitos propriamente ditos; aprender sobre ciência refere-se à compreensão das questões importantes da filosofia, história e metodologias da ciência, ou seja, diz respeito à compreensão dos processos que dão origem ao conhecimento científico; e aprender como fazer ciência refere-se à capacidade do sujeito para participar e realizar atividades que levam ao desenvolvimento do conhecimento científico, isto é, tornar-se parte ativa no processo de construção da ciência.

Um ensino baseado na manipulação e exploração de modelos poderá ser uma das soluções para a reestruturação dos modelos mentais incongruentes com os modelos curriculares. Dada a exigência conceptual dos modelos curriculares, é necessário o recurso à modelação por parte do professor, na tentativa de facilitar a mediação didática necessária à reconceptualização dos modelos mentais dos estudantes.

O professor, enquanto educador, propõe o conhecimento, ao invés de transmiti-lo sem a participação do estudante. Desta forma, o aluno passa a ser parte ativa do processo de construção do conhecimento. A utilização de diferentes metodologias facilita a aprendizagem dos conteúdos que adquirem significado (Alves 2014), havendo assim a restruturação dos seus modelos mentais.

\section{Metodologia}

Com este trabalho pretendemos obter alguns indicadores relativamente à relevância atribuída à modelação, enquanto metodologia usada na lecionação de temáticas de riscos naturais, analisando a existência ou não de referências a atividades de modelação, em unidades curriculares onde são lecionadas as temáticas enunciadas. Para isso, foram recolhidas e analisadas as fichas das unidades curriculares do primeiro ciclo de estudos (licenciatura), que contemplam temáticas de riscos naturais, ministradas em universidades públicas portuguesas e inseridas em cursos com forte componente de geociências.

Após uma pesquisa prévia, foram recolhidas e consideradas para análise oito fichas de unidades curriculares, que compõem os planos de estudos de oito licenciaturas, pertencentes a sete universidades públicas portuguesas. A maioria das fichas analisadas é referente a unidades curriculares semestrais do ano letivo de 2013/2014. No entanto, algumas das fichas cedidas são relativas ao ano letivo de 2012/2013, mas foi garantida a não existência de alterações no conteúdo da ficha da unidade curricular. Posteriormente todas as fichas das unidades curriculares foram analisadas, com base numa grade de análise construída e validada para o efeito por três especialistas com experiência na docência do ensino superior.

Este instrumento possui seis categorias de análise: finalidades; conteúdo conceptual; conteúdo procedimental; conteúdo atitudinal; metodologias 
e avaliação, e cada uma das categorias enunciadas divide-se em várias subcategorias, definidas de acordo com as especificações das fichas das unidades curriculares analisadas, de forma a facilitar a recolha e sistematização das informações.

$\mathrm{Na}$ categoria finalidades pretende-se analisar os objetivos definidos para as unidades curriculares nas várias instituições do ensino superior em estudo. Neste caso, as subcategorias definidas são: desenvolver conhecimentos sobre os riscos naturais/geológicos, promover ações de gestão de recursos, prever e minimizar riscos $\mathrm{e}$, analisar e investigar soluções para problemas associados a situações de risco. Relativamente à categoria conteúdo conceptual as subcategorias definidas dizem respeito aos conteúdos científicos que se espera que os estudantes desenvolvam, nomeadamente: definir risco, perigo e vulnerabilidade, efetuar a avaliação e gestão do risco, analisar os efeitos dos sismos em edifícios e outras estruturas e, prever e prevenir riscos, ações de segurança.

O conteúdo procedimental pretende recolher informações sobre os conhecimentos que os estudantes devem desenvolver relativamente aos processos e métodos científicos, incluindo a utilização de técnicas/software específicas para avaliação e gestão do risco, desenvolver planos de gestão de risco e, analisar, pesquisar e sintetizar informação. Também se considerou importante recolher informações sobre os conteúdos atitudinais que correspondem às atitudes e aos valores que se pretende que os estudantes desenvolvam: desenvolver valores inerentes ao trabatho individual e colaborativo e, desenvolver autonomia e capacidade de organização na gestão de tarefas e do tempo.

No que diz respeito à metodologia, as subcategorias definidas correspondem às principais metodologias enunciadas nas fichas das unidades curriculares analisadas: ensino Tradicional (apresentações PowerPoint ${ }^{\circledR}$, resolução de exercícios, leitura de textos e artigos), modelação (elaboração de modelos), resolução de problemas (análise e discussão de situações-problema), saídas/trabalho de campo e, desenvolvimentos de projetos/trabalhos em grupo. Finalmente, considerou-se pertinente analisar também o tipo de avaliação contemplada nas fichas das unidades curriculares, uma vez que esta deve ser adaptada ao tipo de metodologias adotadas no ensino das unidades curriculares. Desta forma, o tipo de avaliação contemplada consiste em testes/exame final, trabalhos em grupo e, realização de relatórios de atividades.

\section{Apresentação e discussão dos resultados}

Após a seleção das unidades curriculares para análise, de acordo com os critérios estabelecidos, ou seja, unidades curriculares que contemplassem temáticas relacionadas com os riscos naturais e que integrassem cursos de licenciatura, procedeu-se à análise de conteúdo das fichas das unidades curriculares, uma vez que correspondem a documentos oficiais que devem apresentar, ainda que de forma resumida, os objetivos e pressupostos da respetiva unidade curricular a que pertencem.

Os resultados obtidos através dessa análise foram organizados e encontram-se sintetizados na tabela 1, que corresponde a um check-list, estando assinaladas com um $\mathrm{X}$ todas as unidades curriculares cuja ficha contempla cada uma das subcategorias de análise.

Através da análise de cada uma das categorias apresentadas na tabela 1, verifica-se que, relativamente às Finalidades, a maioria das unidades curriculares $(\mathrm{f}=7)$ refere o desenvolvimento de conhecimentos sobre os riscos naturais/geológicos como finalidade da unidade curricular. Contudo, constata-se que algumas fichas das unidades curriculares $(f=4)$ referem também a promoção de ações de gestão de recursos, previsão e minimização de riscos e a análise e investigação de soluções para problemas associados a situações de risco como finalidades das unidades curriculares analisadas.

No que diz respeito ao conteúdo conceptual, 5 unidades curriculares $(\mathrm{f}=5)$ contemplam a definição de risco, perigo e vulnerabilidade e a previsão e prevenção de riscos, açôes de segurança como conteúdos a serem lecionados. Atendendo ao facto de terem sido analisadas unidades curriculares onde são lecionadas temáticas de riscos naturais, era de prever que os conteúdos mais importantes a serem abordados se referissem à noção de risco, perigo e vulnerabilidade, não esquecendo outros assuntos relacionados. Outros conteúdos são também referidos, como a avaliação e gestão do risco e, o efeito dos sismos em edifícios e outras estruturas, sendo que o último apenas é enunciado numa das fichas da unidades curriculares analisadas. O tipo de conteúdos enunciados espelha um pouco os propósitos principais destas unidades curriculares, passando esses objetivos pela sustentabilidade, e a capacidade de prever, prevenir e minimizar os danos causados por estes fenómenos naturais.

Em termos procedimentais, poucas fichas das unidades curriculares fazem referência aos conteúdos que devem ser abordados nesta categoria, mas ainda assim verifica-se que 3 utilizam técnicas/software 
Tabela 1 - Grelha de análise das fichas das unidades curriculares. $(n=8)$

\begin{tabular}{|c|c|c|c|c|c|c|c|c|c|}
\hline \multirow{2}{*}{ Categoria } & \multirow{2}{*}{ Subcategoria } & \multicolumn{8}{|c|}{ Unidades Curriculares } \\
\hline & & U1 & U2 & U3 & U4 & U5 & U6 & U7 & U8 \\
\hline \multirow{3}{*}{ Finalidades } & $\begin{array}{l}\text { Desenvolver conhecimentos sobre } \\
\text { os riscos naturais/ geológicos }\end{array}$ & $\mathrm{X}$ & $\mathrm{X}$ & $\mathrm{X}$ & $\mathrm{X}$ & $\mathrm{X}$ & - & $\mathrm{X}$ & $\mathrm{X}$ \\
\hline & $\begin{array}{l}\text { Promover ações de gestão de } \\
\text { recursos, prever e minimizar riscos }\end{array}$ & - & $\mathrm{X}$ & $\mathrm{X}$ & - & - & $\mathrm{X}$ & - & $\mathrm{X}$ \\
\hline & $\begin{array}{l}\text { Analisar e investigar soluções para } \\
\text { problemas associados a situações de } \\
\text { risco }\end{array}$ & $\mathrm{X}$ & - & - & - & $\mathrm{X}$ & $\mathrm{X}$ & $\mathrm{X}$ & - \\
\hline \multirow{4}{*}{$\begin{array}{l}\text { Conteúdo } \\
\text { Conceptual }\end{array}$} & $\begin{array}{l}\text { Definir risco, perigo e } \\
\text { vulnerabilidade }\end{array}$ & $\mathrm{X}$ & - & - & $\mathrm{X}$ & $\mathrm{X}$ & $\mathrm{X}$ & $\mathrm{X}$ & - \\
\hline & Efetuar a avaliação e gestão do risco & $\mathrm{X}$ & $\mathrm{X}$ & - & $\mathrm{X}$ & - & - & - & $\mathrm{X}$ \\
\hline & $\begin{array}{l}\text { Analisar os efeitos dos sismos em } \\
\text { edifícios e outras estruturas }\end{array}$ & - & - & - & $\mathrm{X}$ & - & - & - & - \\
\hline & $\begin{array}{l}\text { Prever e prevenir riscos, ações de } \\
\text { segurança }\end{array}$ & - & - & $\mathrm{X}$ & $\mathrm{X}$ & $\mathrm{X}$ & - & $\mathrm{X}$ & $\mathrm{X}$ \\
\hline \multirow{3}{*}{$\begin{array}{l}\text { Conteúdo } \\
\text { Procedimental }\end{array}$} & $\begin{array}{l}\text { Utilizar técnicas/software específicas } \\
\text { para avaliação e gestão do risco }\end{array}$ & - & $\mathrm{X}$ & - & - & - & $\mathrm{X}$ & $\mathrm{X}$ & - \\
\hline & $\begin{array}{l}\text { Desenvolver planos de gestão de } \\
\text { risco }\end{array}$ & $\mathrm{X}$ & - & - & - & - & - & - & - \\
\hline & $\begin{array}{l}\text { Analisar, pesquisar e sintetizar } \\
\text { informação }\end{array}$ & $\mathrm{X}$ & - & $\mathrm{X}$ & - & - & $\mathrm{X}$ & - & - \\
\hline \multirow{2}{*}{$\begin{array}{l}\text { Conteúdo } \\
\text { Atitudinal }\end{array}$} & $\begin{array}{l}\text { Desenvolver valores inerentes ao } \\
\text { trabalho individual e colaborativo }\end{array}$ & $\mathrm{X}$ & - & - & - & - & $\mathrm{X}$ & - & $\mathrm{X}$ \\
\hline & $\begin{array}{l}\text { Desenvolver autonomia e } \\
\text { capacidade de organização na gestão } \\
\text { de tarefas e do tempo }\end{array}$ & $\mathrm{X}$ & - & $X$ & - & - & $\mathrm{X}$ & - & $\mathrm{X}$ \\
\hline \multirow{6}{*}{ Metodologia } & $\begin{array}{l}\text { Ensino Tradicional (apresentações } \\
\text { PowerPoint }{ }^{\circledR}, \text { resolução de } \\
\text { exercícios, leitura de textos e artigos) }\end{array}$ & - & - & $\mathrm{X}$ & $\mathrm{X}$ & $\mathrm{X}$ & $X$ & $\mathrm{X}$ & - \\
\hline & Modelação (elaboração de modelos) & - & - & - & - & - & - & - & - \\
\hline & $\begin{array}{l}\text { Resolução de Problemas (análise e } \\
\text { discussão de situações-problema) }\end{array}$ & $X$ & - & - & $\mathrm{X}$ & - & - & - & - \\
\hline & Saídas/Trabalho de campo & - & $\mathrm{X}$ & $\mathrm{X}$ & $\mathrm{X}$ & - & $\mathrm{X}$ & - & - \\
\hline & $\begin{array}{l}\text { Desenvolvimento de projetos/ } \\
\text { Trabalhos em grupo }\end{array}$ & - & $\mathrm{X}$ & $\mathrm{X}$ & - & - & $\mathrm{X}$ & - & $\mathrm{X}$ \\
\hline & Testes/ Exame Final & - & $\mathrm{X}$ & $X$ & $\mathrm{X}$ & $\mathrm{X}$ & $\mathrm{X}$ & $\mathrm{X}$ & $\mathrm{X}$ \\
\hline \multirow[t]{2}{*}{ Avaliação } & Trabalhos em grupo & $\mathrm{X}$ & - & - & - & - & $\mathrm{X}$ & - & $\mathrm{X}$ \\
\hline & Relatórios de atividades & - & $\mathrm{X}$ & $\mathrm{X}$ & - & - & $\mathrm{X}$ & $\mathrm{X}$ & $X$ \\
\hline
\end{tabular}


específicas para avaliação e gestão do risco e respetivos procedimentos implícitos e, referem a análise, pesquisa e sintetização de informação como procedimentos igualmente relevantes. Também a nível do conteúdo atitudinal a informação disponível nas fichas das unidades curriculares é vaga, referindo-se geralmente ao desenvolvimento da autonomia dos estudantes, a capacidade de realizar trabalhos em grupo e várias atitudes inerentes ao desenvolvimento do trabalho colaborativo, que correspondem a atitudes que se espera que os estudantes desenvolvam ao longo do seu processo de construção do conhecimento. Deste modo, 4 fichas das unidades curriculares $(\mathrm{f}=4)$ referem o desenvolvimento da autonomia e a capacidade de organização na gestão de tarefas e do tempo e 3 unidades curriculares $(\mathrm{f}=3$ ) referem o desenvolvimento de valores inerentes ao trabalho individual e colaborativo como atitudes a desenvolver.

Relativamente à categoria referente à metodologia, verifica-se que a maioria das fichas das unidades curriculares $(\mathrm{f}=5)$ contempla a existência de um ensino tradicional, que compreende o recurso a apresentações PowerPoint ${ }^{\circledast}$ resolução de exercícios, leitura de textos e artigos científicos. Além desta metodologia, há algumas fichas de unidades curriculares $(\mathrm{f}=4)$ que referem um ensino baseado em saídas para o exterior elou o trabalho de campo, assim como o desenvolvimento de projetos e/ou a realização de trabalhos em grupo. Contudo, nenhuma das fichas das unidades curriculares em análise faz referência a um ensino investigativo baseado em modelos.

A avaliação das unidades curriculares em análise ocorre predominantemente $(\mathrm{f}=7)$ através da realização de testes realizados durante o período letivo, ou através de um exame final. Além deste instrumento de avaliação são referidos outros como a realização de relatórios de atividades ( $\mathrm{f}=6$ ) e a elaboração de trabalhos em grupo $(\mathrm{f}=3)$. É notória a diversidade de instrumentos de avaliação dos estudantes, não se baseando apenas na realização de testes ou exames para avaliação do conhecimento conceptual, aplicando diferentes tipos de avaliação consoante a metodologia que mais valorizam.

\section{Conclusões}

De acordo com os resultados apresentados anteriormente é possível concluir que nenhuma das fichas das unidades curriculares em análise contempla o recurso à modelação como metodologia de ensino de temáticas de riscos naturais. Também se verifica que a lecionação das temáticas relativas aos riscos naturais ocorre através de um ensino predominantemente tradicional, que é apoiado em apresentações PowerPoint ${ }^{\circledR}$ e na leitura e discussão de artigos científicos, sendo de notar, a ausência de referência a qualquer tipo de exploração dos artigos científicos para além da sua leitura.

O conteúdo das fichas das unidades curriculares pode, até certo ponto, ser limitado e não representar a totalidade de conteúdos que são explorados nas unidades curriculares a que correspondem. Isto deve-se ao facto de o seu preenchimento nos websites das várias universidades estar condicionado a um número de carateres limitado, facto que pode ter impossibilitado a descrição pormenorizada de algumas dimensões em análise. Contudo, as fichas das unidades curriculares constituem os documentos oficiais que estão geralmente disponíveis para consulta dos estudantes, e por isso, devem espelhar os principais objetivos e conteúdos da unidade curricular. Assim, consideramos que os aspetos mais relevantes são sempre mencionados e neles não se incluiu um ensino com recurso à modelação. Pelo exposto, os autores consideram que é necessário intervir ao nível das metodologias de ensino, na tentativa de se valorizar o papel dos modelos e da modelação no ensino superior português para lecionação de temáticas de riscos naturais. Esta intervenção deverá envolver fortemente os docentes, pois estes devem desenvolver competências para compreenderem como ocorre o processo de construção dos modelos mentais dos estudantes e como o uso de modelos em sala de aula facilita a reestruturação desses modelos mentais. Desta forma, é imprescindível que conheçam a natureza dos modelos, que percebam como estes devem ser introduzidos na sala de aula, e principalmente tenham a capacidade de desenvolver modelos que possam ser usados no ensino (Justi \& Gilbert 2002b).

Futuramente, importa analisar se o recurso aos modelos se adequa e auxilia a reestruturação dos modelos mentais dos estudantes do ensino superior, contribuindo para o desenvolvimento de uma aprendizagem significa.

\section{Agradecimentos}

Os autores agradecem o apoio prestado pelo Instituto de Ciências da Terra (ICT), sob contrato com a FCT - Fundação Portuguesa para a Ciência e Tecnologia. 
elaboración de modelos. Enseñanza de las Ciencias, 24(2):173-184.

\section{Referências}

Alves F.M. 2014. Enriquecendo o ensino de ciências através do uso de modelos didáticos: uma abordagem com o sistema circulatório humano. II Encontro de Pesquisa em Ensino das Ciências e Matemática: questões atuais. 1(1):32-34.

Barbosa J.C. 2009. Modelagem e Modelos Matemáticos na Educação Científica. Revista de Educação em Ciência e Tecnologia, 2(2):69-85.

Bolacha E., Deus H.M., Fonseca P.E. 2011. The concept of analogue modelling in Geology: an approach to mountain building. In Proceedings of the 9th ESERA Conference. Univ. Lyon, France.

Brandstetter T. 2011. Time Machines: Models Experiments in Geology. Centaurus, 53: 135-145.

Clement J. 2000. Model based learning as a key research area for science education. Intern. J. Sci. Educ., 22(9):1041-1053.

Deus H.M., Bolacha E., Vasconcelos C., Fonseca P.E. 2011. Analogue modelling to understand geological phenomena. In Proceedings of the GeoSciEd VI. Joahnnesburg, South Africa.

Gobert J.D., Buckley B.C. 2000. Introduction to model-based teaching and learning in science education. Intern. J. Sci. Educ., 22(9):891-894.

Graveleau F., Malavieille J., Dominguez S. 2012. Experimental modelling of orogenic wedges: A review. Tectonophysics, 1-66.

Greca I.M., Moreira M.A. 2000. Mental models, conceptual models, and Modeling. Intern. J. Sci. Educ., 22(1):1-11.

Hodson D. 1992. In search of a meaningful relationship: an exploration of some issues relating to integration in science and science education. Intern. J. Sci. Educ., 14:541-562.

Johnson-Laird P.N. 1983. Mental Models. Towards a Cognitive Science of Language, Inference and Consciousness, Cambridge: Harvard Univ. Press, 126-146.

Justi R. 2006. La enseñanza de ciencias basada en la
Justi R.S., Gilbert J.K. 2002a. Modelling, teachers' views on the nature of modelling, and implications for the education of modellers. Intern. J. Sci. Educ., 24(4):369-387.

Justi R.S., Gilbert J.K. 2002b. Science teachers' knowledge about and attitudes towards the use of models and modelling in learning science. Intern.J. Sci. Educ., 24(12): 1273-1292.

Krapas S., Queiroz G., Colinvaux D., Franco C. 1997. Modelos: Uma análise de sentidos na literatura de pesquisa em ensino de ciências. Invest. Ens. Ciências, 2(3): 185-205.

Louca L.T., Zacharia Z.C., Constantinou C.P. 2011. In Quest of Productive Modeling-Based Learning Discourse in Elementary School Science. $J$. Res .Sci. Teaching, 48(8): 919-951.

Moreira M.A. 1996. Modelos Mentais. Investi. Ens. Ciências, 1(3): 193-232.

Moreira M.A. 2014. Modelos científicos, modelos mentais, modelagem computacional e modelagem matemática: aspectos epistemológicos e implicações para o ensino. Rev. Bras. Ens. Ciênc. Tecnol., 7(2):1-20.

Moutinho S., Torres J., Almeida A., Vasconcelos C. 2013. Portuguese teachers' views about geosciences models. IX Congreso Internacional sobre Investigación en Didáctica de las Ciencias, p. 2430-2435.

Palmero M.L.R. 2008. La Teoría de los Modelos Mentales de Johnson-Laird. In: Palmero M.L.R., Moreira M.A., Sahelices M.C.C. e Greca I.M. eds. 2008. La Teoría del Aprendizaje Significativo en la perspectiva de la Psicología Cognitiva, Barcelona: Ed. Octaedro, p.46-87.

Torres J., Vasconcelos C. 2014. Os modelos e a modelação na formação inicial de professores de Biologia e de Geologia. Comunicações Geológicas. 101:1-4.

Treagust D.F., Chittleborough G., Mamiala T.L. 2002. Students' understanding of the role of scientific models in learning science. Intern. J. Sci. Educ., 24(4):357-368.

Resumo: 0 recurso à modelação como metodologia de ensino é relevante no processo de recriação e simulação de fenómenos naturais, nomeadamente fenómenos geológicos e ambientais. Pretendendo-se verificar se o recurso à modelação é uma metodologia significativa no sucesso da aprendizagem dos estudantes de riscos naturais do ensino superior português, foram analisadas as fichas das unidades curriculares que abordam temáticas de riscos naturais nas universidades públicas portuguesas, tendo-se optado por selecionar apenas unidades curriculares do primeiro ciclo de estudos - licenciatura. No total foram analisadas, com recurso a uma grade de análise, oito fichas de unidades curriculares, ministradas em sete universidades públicas portuguesas. As evidências encontradas indicam que nenhum dos documentos em análise contempla a utilização da modelação como metodologia de ensino de temáticas de riscos naturais, predominando um ensino essencialmente tradicional, sobressaindo a necessidade de intervenção ao nível das metodologias de ensino valorizando-se a manipulação e a exploração de modelos no ensino superior.

Palavras-chave: Modelação; ensino superior; riscos naturais; fichas de unidades curriculares. 